\title{
A Microsoft HoloLens Mixed Reality Surgical Simulator for Patient-Specific Hip Arthroplasty Training
}

\author{
Giuseppe Turini ${ }^{1,2}$, Sara Condino ${ }^{2,3(\varangle)}$, Paolo Domenico Parchi ${ }^{2,4}$, Maria Rosanna \\ Viglialoro $^{2}$, Nicola Piolanti ${ }^{4}$, Marco Gesi ${ }^{5,6}$, Mauro Ferrari ${ }^{2}$, Vincenzo Ferrari ${ }^{2,3}$ \\ ${ }^{1}$ Computer Science Department, Kettering University, Flint, MI, USA \\ gturini@kettering.edu \\ ${ }^{2}$ EndoCAS Center, Department of Translational Research and of New Surgical and Medical \\ Technologies, University of Pisa, Pisa, Italy. \\ \{sara.condino, rosanna.viglialoro, \\ vincenzo.ferrari\} @endocas.unipi.it \\ ${ }^{3}$ Department of Information Engineering, University of Pisa, Pisa, Italy. \\ ${ }^{4} 1^{\text {st }}$ Orthopaedic and Traumatology Division, Department of Translational Research and of \\ New Surgical and Medical Technologies, University of Pisa, Pisa, Italy. \\ paolo.parchi@unipi.it, nicpio@hotmail.it \\ ${ }^{5}$ Department of Translational Research and of New Surgical and Medical Technologies, \\ University of Pisa, Pisa, Italy. \\ \{marco.gesi, mauro.ferrari\} @ med.unipi.it \\ ${ }^{6}$ Center for Rehabilitative Medicine "Sport and Anatomy", University of Pisa, Pisa, Italy.
}

\begin{abstract}
Surgical simulation can offer novice surgeons an opportunity to practice skills outside the operating theatre in a safe controlled environment. According to literature evidence, nowadays there are very few training simulators available for Hip Arthroplasty (HA).

In a previous study we have presented a physical simulator based on a lower torso phantom including a patient-specific hemi-pelvis replica embedded in a soft synthetic foam. This work explores the use of Microsoft HoloLens technology to enrich the physical patient-specific simulation with the implementation of wearable mixed reality functionalities. Our HA multimodal simulator based on mixed reality using the HoloLens is described by illustrating the overall system, and by summarizing the main phases of the design and development.

Finally, we present a preliminary qualitative study with seven subjects ( 5 medical students, and 2 orthopedic surgeons) showing encouraging results that suggest the suitability of the HoloLens for the proposed application. However, further studies need to be conducted to perform a quantitative test of the registration accuracy of the virtual content, and to confirm qualitative results in a larger cohort of subjects.
\end{abstract}

Keywords: Surgical Simulation, Augmented Reality, Microsoft HoloLens, Hip arthroplasty.

adfa, p. 1, 2011.

(c) Springer-Verlag Berlin Heidelberg 2011 


\section{$1 \quad$ Introduction}

Hip arthroplasty (HA) is a surgical procedure which involves replacing a damaged hip joint with a prosthetic implant to reduce pain and improve mobility. HA is one of the most widely performed procedures in orthopedic practice and it is considered to be one of the most successful orthopedic interventions of the $20^{\text {th }}$ century. However primary and revision total hip arthroplasty (THA) have been ranked third and fourth among the orthopedic interventions accounted for the greatest share of adverse events and excess hospital stay [1]. The risk of HA complications is strongly related to the surgeon's case volume. According to a research study [2], surgeons should perform at least 35 THA procedures annually to reduce the risk of complications. Another study [3] focalized on the anterior approach, shows that 50 or more procedures are required before reaching the performance (in terms of revision rate) of surgeons with an experience of 100 or more cases.

In this context, surgical simulation could play a pivotal role, offering novices an opportunity to practice skills outside the operating theatre, in a safe controlled environment. Several techniques of simulation are today available including: physical, virtual reality (VR), and hybrid simulation. This latter is based on the combination of computer simulation with synthetic models of the anatomy. The physical models can mimic the mechanical properties of human tissue [5-7] offering the learner the possibility to practice skills using real surgical instruments; whereas computer graphics enables the visualization and animation of virtual content, enhancing the surgical simulation. Thus, hybrid simulators can guarantee a realistic haptic feedback, overcoming the limitations of current VR technologies which are still unable to accurately model in real-time the instruments-anatomy interactions. At the same time, augmented reality (AR) features allow the guidance of the trainee during the execution of surgical tasks, and the visualization of hidden anatomical structures as virtual organs [8-13].

According to a recent review study [14], nowadays there are very few training simulators available for HA. Indeed, even if VR systems have been developed for planning purposes, there are not many simulators aimed at training.

In a previous work [15] we have presented the "HipSim", a physical simulator based on a lower torso phantom including a patient-specific hemi-pelvis replica embedded in a soft synthetic foam. The "HipSim" was validated by 13 orthopedic surgeons and considered a realistic and useful learning tool.

This work explores the use of Microsoft HoloLens technology to enrich the physical patient-specific simulation with the implementation of wearable mixed reality functionalities.

The Microsoft HoloLens, one of the most advanced head-mounted displays (HMDs) available today, can provide users hands-free access to complex data, and an unobstructed view of the real environment. Recent literature works have addressed the usage of the HoloLens in the operating room to improve decision-making and surgical workflow [16], remote supervision and annotation, 3D image viewing and manipulation, telepathology in a mixed-reality environment, and real-time pathologyradiology correlation [17]. 
In this paper we present the design and development of a HA multimodal simulator based on mixed reality using the Microsoft HoloLens: combining patient-specific synthetic replicas, AR features, and audio technology.

\section{$2 \quad$ Material and Methods}

The following paragraphs briefly describe the HoloLens Technology, and the main steps for the design of the proposed Mixed Reality Surgical Simulator.

\subsection{Head-Mounted Display: Overview of the HoloLens Technology}

The HoloLens, is an optical see-through (OST) HMD: it allows superposition of digital information onto the real-world view, while optically maintaining see-through view which assures that visual and proprioception information is synchronized. This HMD, was chosen for the development of our HA multimodal simulator since it provides significant benefits over other commercial HMDs from human factors and ergonomics standpoints [18] and integrates important functionalities for an immersive and interactive simulation experience.

The HoloLens technology is based on an undisclosed Intel 32-bit processor, with a custom-built Microsoft Holographic Processing Unit (HPU 1.0) which supports Universal Windows Platform (UWP) apps. It is equipped with 2 GB of RAM (1GB for $\mathrm{CPU}$, and 1GB for HPU) and 64 GB of flash memory; it features network connectivity via Wi-Fi 802.11ac and Bluetooth 4.1 LE wireless technology. The HPU processes data from multiple sensors: 4 "environment understanding cameras"; 1 depth camera; and 1 world-facing photo/video camera (2 MP); 1 inertial measurement unit (IMU) to track head movements; 4 microphones; and 1 ambient light sensor.

Thanks to these technologies the HoloLens provides distinctive features such as: spatial mapping, interactions by means of gaze (estimated from head tracking), hand gesture, and voice commands. The speakers integrated into the headset enable binaural audio to simulate effects such as spatial sounds within the user's environment.

For all these reasons, HoloLens can be considered a perfect candidate for the implementation of mixed-reality surgical simulators. However, some technical issues have to be considered, such as: restricted overlay field of view (FOV) (only about $35^{\circ}$ ); vergence-accommodation conflict; mismatch between the focal distance of real and virtual objects (HoloLens features a fixed focal length of $2.0 \mathrm{~m}$ ); difficulties in handling occlusions between the real and virtual contents. For a complete overview of these issues refer to [19]. Finally, the weight of $579 \mathrm{~g}$ can make it uncomfortable to wear for prolonged periods of time.

For all these reasons, tests should be performed to evaluate the usability of the proposed HDM for our specific scenario: orthopedic open surgery. 


\subsection{Design of the Mixed Reality Surgical Simulator}

Virtual and Physical Contents. The development of the simulator started from the extraction of the anatomical components from a real radiological computed tomography (CT) data set. CT images were processed using a semi-automatic tool, the EndoCAS Segmentation Pipeline, integrated in the open source software ITK-SNAP 1.5. Then mesh optimization stages (artifacts removal, holes filling, simplification, and filtering) were performed using the open source softwares MeshLab and Blender. Segmented anatomical models include: pelvis, hip bones, sacrum, coccyx, femoral heads, principal muscles around the hip joint (such as: gluteal muscles, piriformis, inferior gemellus, superior gemellus, obturator internus).

Rapid prototyping (Dimension Elite 3D Printer) and casting techniques were used to fabricate synthetic replicas of anatomical parts for which it is important to guarantee the surgeon the possibility of physical interaction. These include: a soft replica of the pelvis with a skin-like covering which allows an accurate simulation of palpation and surgical incision; a model of the acetabulum in acrylonitrile butadiene styrene (ABS), a material commonly used for the manufacturing of bone replicas for surgical drilling simulation. The main stages of the manufacturing of the physical simulator are illustrated in Figure 1.

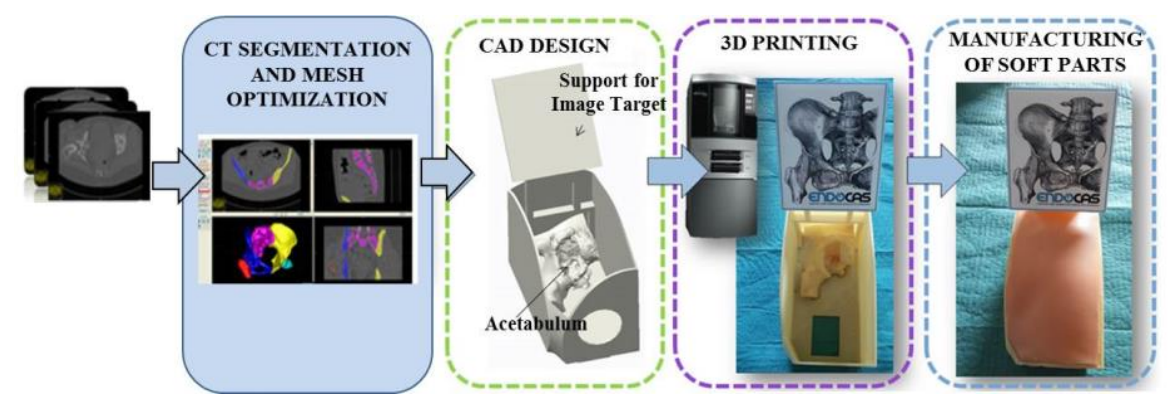

Fig. 1. The manufacturing of the physical simulator, from left to right: the CT data set, the segmentation stage, the $\mathrm{CAD}$ design and 3D printing for the rapid prototyping of the synthetic anatomy, and the complete physical simulator including the support for a Vuforia Image Target used to register the virtual content with the mannequin.

Other segmented anatomical structures, important to increase the anatomical knowledge of the user trainee, are instead visualized as virtual organs (virtual content). This virtual anatomy is enriched with:

- a collection of radiological images, which present the surgical case to the learner at the beginning of the simulation (see Figure 2, and the Medical Image Navigator module in Figure 3);

- information from a simulated planning phase with the 3D Hip Plugin [15]: a pair of "viewfinders", and a virtual line showing the surgeon the optimal trajectory to insert the surgical reamer (see Figure 3 and Figure 4). 
The detection and tracking functionalities offered by the Vuforia SDK were used for the real-time registration of the virtual content to the real scene. At this end, the physical environment includes the support for a Vuforia Image Target, which was rigidly anchored to the bone synthetic replica (see Figure 1).

Software Implementation. Unity3D (5.6.1f) was used to implement the software application, using the MixedRealityToolkit (2017.1.2), a freely available collection of C\# scripts which allows an easy and fast development of AR applications. The developed mixed reality app allows the user to interact with the virtual content by means of: gaze (estimated from the position and orientation of the user's head), HoloLens gestures (Air Tap and Bloom), and voice commands (detected by Microsoft Cortana speech recognition on the HoloLens). A virtual menu with toggle buttons has been implemented to allow the user to control the visualization of the virtual content (pelvis, bones, muscles, pre-operative plan).

Operating room sounds effects (including voices and hospital machines sounds) are added to improve immersion and increase the realism of the simulation.

A Medical Image Navigator module (Figure 2) was implemented for the visualization of radiological images. This navigator allows: the anchoring of the radiological images collection in a user-defined position (using the Air Tap gesture), and the switching to the next image in the collection (using the voice command "Next").
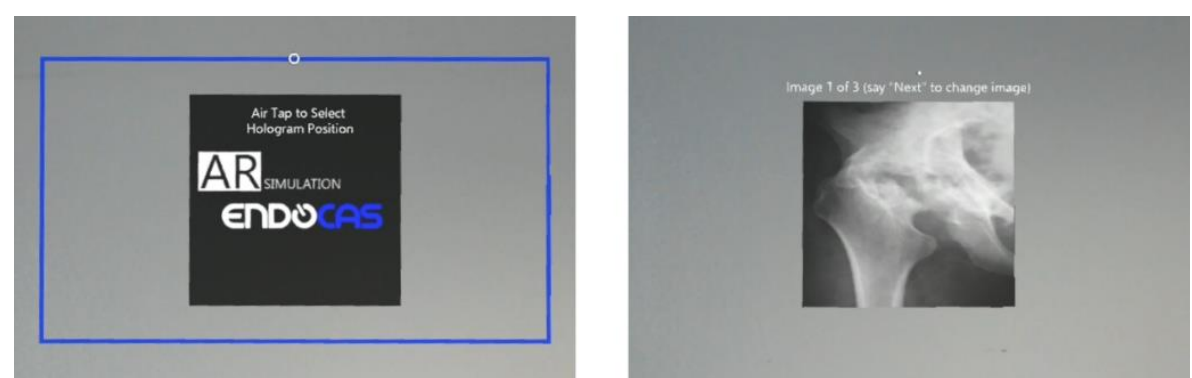

Fig. 2. Two "screenshots" of the Medical Image Navigator module: the opening screen to allow the user to anchor the mixed reality window using the Air Tap gesture (left), and the first medical image of a collection of 3 that the user can navigate using voice commands (right).

Figure 3 shows the system diagram of the "HipSim" mixed reality application illustrating all the interactions between the hardware and software modules, and showing all the user-interactions involved. 


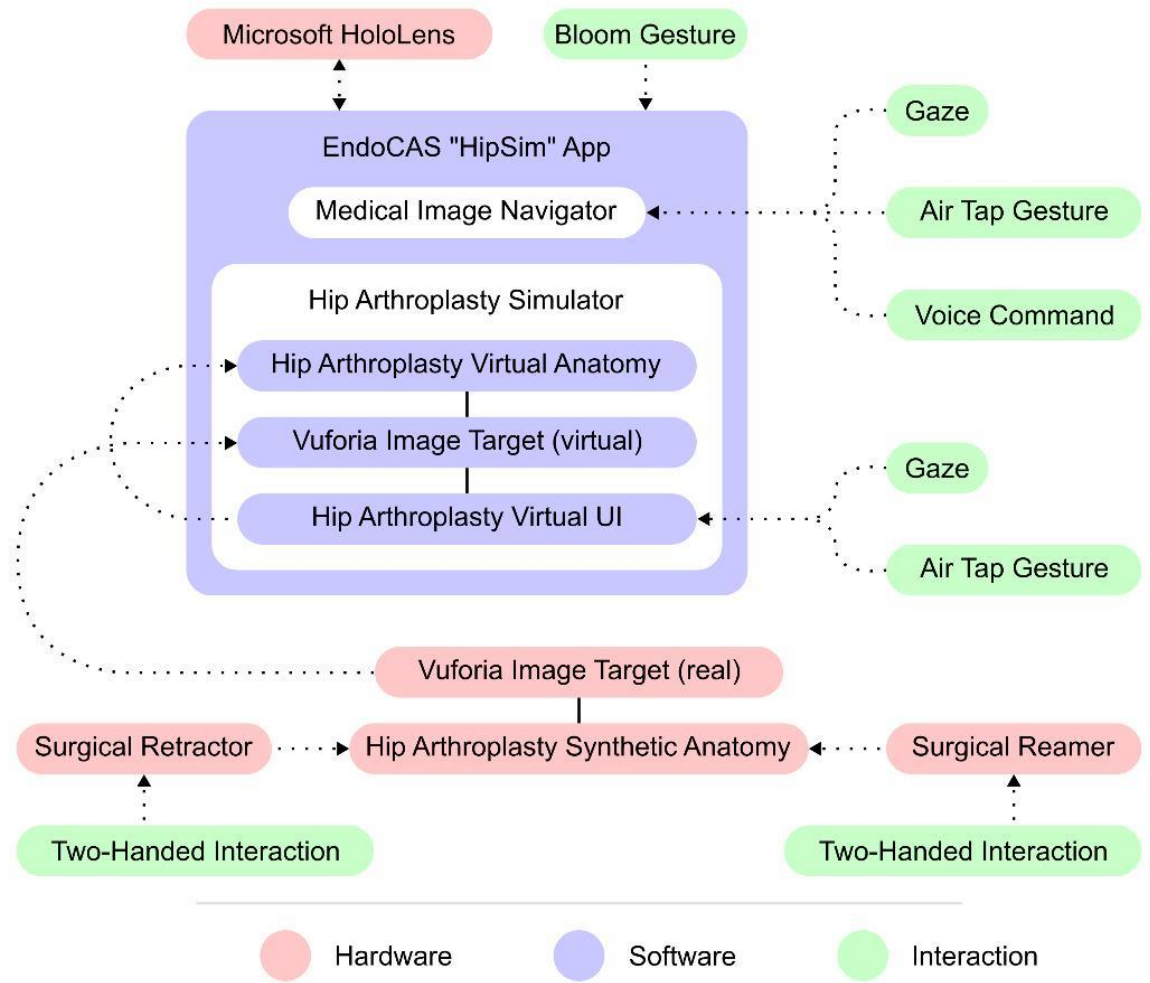

Fig. 3. Scheme of the "HipSim" mixed reality application for the Microsoft HoloLens, illustrating all the interactions between the hardware and software modules (dotted lines), the different types of interactions (voice, gestures etc.), as well as the "rigid" geometrical transformations (solid lines) between some physical and virtual modules.

\section{Evaluation of the Mixed Reality Surgical Simulator}

Seven (7) subjects, two (2) orthopedic surgeons and five (5) medical students of the University of Pisa, were recruited to participate in a preliminary qualitative study of our hip arthroplasty multimodal simulator. None of the participants had any previous experience with the Microsoft HoloLens. Table 1 summarizes the demographic characteristics of the participants. The experimental setup is depicted in Figure 4. 
Table 1. Demographic characteristics of the participants.

\begin{tabular}{|l|c|}
\hline Position held (medical students, orthopedic surgeons). & 5,2 \\
\hline Gender (male, female, non-binary). & $5,2,0$ \\
\hline Age (min, max, mean, STD). & $23,39,30,6$ \\
\hline Vision (10/10 naked eyes, corrected to 10/10 with contact lenses). & 2,5 \\
\hline Experience with AR (none, limited, familiar, experienced). & $3,3,1,0$ \\
\hline Experience with VR (none, limited, familiar, experienced). & $2,4,1,0$ \\
\hline Experience with HoloLens (none, limited, familiar, experienced). & $7,0,0,0$ \\
\hline Color Blindness (no, yes). & 20,0 \\
\hline
\end{tabular}

Each subject performed the calibration of the HoloLens using the Microsoft "Calibration" app, and then was asked to familiarize with gestural controls using the Microsoft "Learn Gestures" app. Finally, each candidate used our HA multimodal simulator for 30 minutes, inspecting the AR content and evaluating: positioning, stability, and visual quality of all the virtual content. More in particular they were instructed to use:

- the Gaze interaction to move the Medical Image Navigator module in the physical space, and to select toggle buttons of the Hip Arthroplasty Virtual UI;

- the voice command "Next" to scroll images of the Medical Image Navigator;

- the Air Tap gesture to anchor the Medical Image Navigator Module in the desired position, and to turn on/off the toggle buttons of the Hip Arthroplasty Virtual UI.

At the end of each trial, the candidate was asked to complete a 5-points Likert questionnaire (from $1=$ strongly disagree, to $5=$ strongly agree) (Table 2 ) grouped under 4 headings: AR Positioning and Time Response, Interaction and Guidance, FOV, and Spatial Sounds.
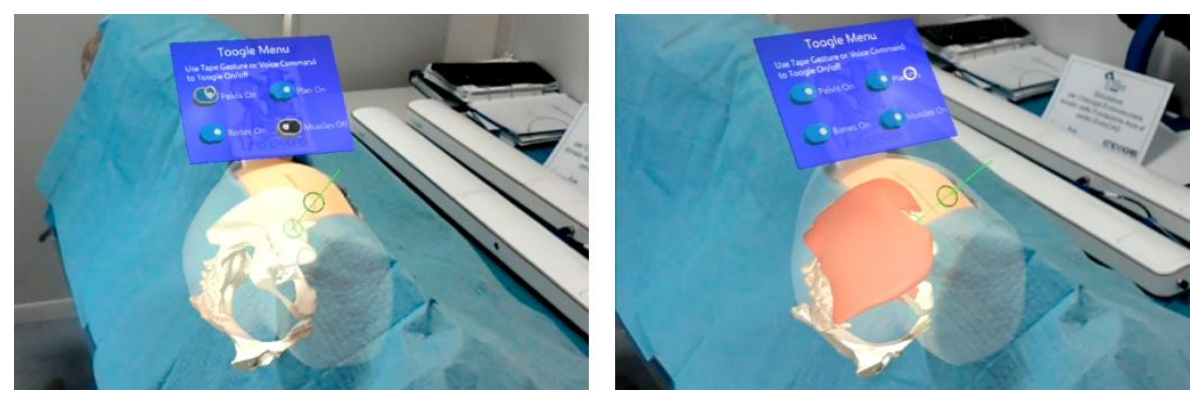

Fig. 4. Two "screenshots" of the Hip Arthroplasty Simulator, showing the virtual user interface the virtual anatomy in two different configurations: the visualization of the bones, pelvis, and preoperative planning (left), with the muscles hidden (deactivated); and the visualization of the complete virtual content (right).

\section{$4 \quad$ Preliminary Results and Discussion}

Table 2 summarizes the results of the preliminary study. The central tendencies of responses to a single Likert item are summarized by using median, with dispersion 
measured by interquartile range. A positive feedback was received about the AR positioning and the time response (first heading), and the suitability of the HoloLens FOV for the proposed application. Participants strongly agreed that Gaze, Gesture, and Voice interactions are easy and intuitive; however, a neutral opinion was expressed about the ease of aligning the surgical instrument to the AR viewfinders. Finally, participants strongly agreed that spatial sounds make the experience more immersive.

Table 2. Likert Questionnaire Results

\begin{tabular}{|c|c|c|c|}
\hline \multicolumn{2}{|r|}{ Questionnaire Items } & Median & IQR-Range \\
\hline \multirow{4}{*}{ 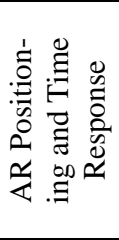 } & The virtual content is correctly aligned to the real objects. & 4.5 & $5-4$ \\
\hline & The positioning of virtual content is stable over the time. & 4 & $5-4$ \\
\hline & $\begin{array}{l}\text { It is easy to perceive the spatial relationships between } \\
\text { real and virtual objects. }\end{array}$ & 4.5 & $5-4$ \\
\hline & $\begin{array}{l}\text { I did not notice latency (lag, delay) between virtual con- } \\
\text { tent and objects real. }\end{array}$ & 4 & $5-4$ \\
\hline \multirow{4}{*}{ 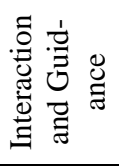 } & Gaze interaction is easy and intuitive. & 5 & $5-4$ \\
\hline & Gesture interactions are easy and intuitive. & 5 & $5-4$ \\
\hline & Voice interactions are easy and intuitive. & 5 & $5-4$ \\
\hline & It is easy to align the surgical tool to the AR viewfinders. & 3.5 & $4.25-2.75$ \\
\hline D & The Field of View (FOV) is adequate for the application. & 4 & $3-4$ \\
\hline 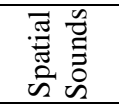 & Spatial sounds make the experience more immersive. & 5 & $5-4$ \\
\hline
\end{tabular}

Obtained results suggest the suitability of the HoloLens for the proposed application. However, further studies need to be conducted to perform a quantitative test of the registration accuracy of the virtual content, and to confirm qualitative results in a larger cohort of subjects.

Acknowledgment. The research leading to these results has been partially supported by the European Project VOSTARS (H2020 Call ICT-29-2016 G.A. 731974)

\section{References}

1. Wolf, B.R., Lu, X., Li, Y., Callaghan, J.J., Cram, P.: Adverse outcomes in hip arthroplasty: long-term trends. The Journal of bone and joint surgery. American volume 94, e103 (2012)

2. Hasegawa, Y., Amano, T.: Surgical Skills Training for Primary Total Hip Arthroplasty. Nagoya J Med Sci 77, 51-57 (2015)

3. de Steiger, R.N., Lorimer, M., Solomon, M.: What is the learning curve for the anterior approach for total hip arthroplasty? Clin Orthop Relat Res 473, 3860-3866 (2015)

4. Carbone, M., Condino, S., Mattei, L., Forte, P., Ferrari, V., Mosca, F.: Anthropomorphic ultrasound elastography phantoms - characterization of silicone materials to build breast elastography phantoms. Conference proceedings : ... Annual International Conference of the IEEE Engineering in Medicine and Biology Society. IEEE Engineering in Medicine and Biology Society. Conference 2012, 492-494 (2012) 
5. Nysaether, J.B., Dorph, E., Rafoss, I., Steen, P.A.: Manikins with human-like chest properties--a new tool for chest compression research. IEEE transactions on bio-medical engineering 55, 2643-2650 (2008)

6. Sparks, J.L., Vavalle, N.A., Kasting, K.E., Long, B., Tanaka, M.L., Sanger, P.A., Schnell, K., Conner-Kerr, T.A.: Use of Silicone Materials to Simulate Tissue Biomechanics as Related to Deep Tissue Injury. Adv Skin Wound Care 28, 59-68 (2015)

7. Botden, S.M., Jakimowicz, J.J.: What is going on in augmented reality simulation in laparoscopic surgery? Surg Endosc 23, 1693-1700 (2009)

8. Carbone, M., Condino, S., Ferrari, V., Ferrari, M., Mosca, F.: Surgical simulators integrating virtual and physical anatomies. In: CEUR Workshop Proceedings, pp. 13-18 (2011)

9. Condino, S., Carbone, M., Ferrari, V., Faggioni, L., Peri, A., Ferrari, M., Mosca, F.: How to build patient-specific synthetic abdominal anatomies. An innovative approach from physical toward hybrid surgical simulators. Int J Med Robot Comp 7, 202-213 (2011)

10. Viglialoro, R., Condino, S., Gesi, M., Ferrari, M., Ferrari, V.: Augmented Reality Simulator for Laparoscopic Cholecystectomy Training. In: De Paolis, L.T., Mongelli, A. (eds.) Augmented and Virtual Reality, pp. 428-433. Springer International Publishing (2014)

11. Ferrari, V., Viglialoro, R.M., Nicoli, P., Cutolo, F., Condino, S., Carbone, M., Siesto, M., Ferrari, M.: Augmented reality visualization of deformable tubular structures for surgical simulation. The international journal of medical robotics + computer assisted surgery : MRCAS (2015)

12. Viglialoro, R., Condino, S., Freschi, C., Cutolo, F., Gesi, M., Ferrari, M., Ferrari, V.: AR visualization of "synthetic Calot's triangle" for training in cholecystectomy. 12th IASTED International Conference on Biomedical Engineering, BioMed 2016 (2016)

13. Vaughan, N., Dubey, V.N., Wainwright, T.W., Middleton, R.G.: A review of virtual reality based training simulators for orthopaedic surgery. Medical Engineering \& Physics 38, 59-71 (2016)

14. Sato, Y., Sasama, T., Sugano, N., Nakahodo, K., Nishii, T., Ozono, K., Yonenobu, K., Ochi, T., Tamura, S.: Intraoperative simulation and planning using a combined acetabular and femoral (CAF) navigation system for total hip replacement. Medical Image Computing and Computer-Assisted Intervention - Miccai 2000 1935, 1114-1125 (2000)

15. Parchi, P., Condino, S., Carbone, M., Gesi, M., Ferrari, V., Ferrari, M., Lisanti, M.: Total hip replacement simulators with virtual planning and physical replica for surgical training and reharsal. In: Proceedings of the 12th IASTED International Conference on Biomedical Engineering, BioMed 2016, pp. 97-101. (Year)

16. Tepper, O.M., Rudy, H.L., Lefkowitz, A., Weimer, K.A., Marks, S.M., Stern, C.S., Garfein, E.S.: Mixed Reality with HoloLens: Where Virtual Reality Meets Augmented Reality in the Operating Room. Plastic and reconstructive surgery 140, 1066-1070 (2017)

17. Hanna, M.G., Ahmed, I., Nine, J., Prajapati, S., Pantanowitz, L.: Augmented Reality Technology Using Microsoft HoloLens in Anatomic Pathology. Archives of pathology \& laboratory medicine (2018)

18. Nicholson, D.: Advances in Human Factors in Cybersecurity: Proceedings of the AHFE 2017 International Conference on Human Factors in Cybersecurity, July 17-21, 2017, The Westin Bonaventure Hotel, Los Angeles, California, USA. Springer International Publishing (2017)

19. Rolland, J.P., Fuchs, H.: Optical versus video see-through mead-mounted displays in medical visualization. Presence-Teleoperators and Virtual Environments 9, 287-309 (2000) 